\title{
Apprendre à " relancer " une activité éducative : La formation professionnelle en situation de travail dans le contexte de l'éducation de l'enfance
}

\author{
Marianne Zogmal, Université de Genève \\ Laurent Filliettaz, Université de Genève \\ Vasiliki Markaki-Lothe, Université Grenoble-Alpes
}

\begin{abstract}
Cet article porte sur l'identification et le développement des compétences professionnelles dans le champ de l'éducation de l'enfance. Dans une perspective interactionnelle, une analyse détaillée étudie les pratiques mobilisées pour apprendre à relancer une activité éducative. L'article présente la notion de relance, avant d'exposer la démarche d'analyse interactionnelle adoptée. L'analyse empirique décrit une activité de relance accomplie par une étudiante en formation et étudie les modalités de participation des différents interactant.e.s qui convergent, progressivement, vers une activité commune. Une focale sur une situation de stage professionnel vise à éclairer les liens entre le phénomène de la relance et les enjeux de la formation professionnelle en situation de travail.
\end{abstract}

\section{Introduction}

Dans l'accueil collectif de la petite enfance, les éducatrices et éducateurs différencient les activités menées auprès des enfants, en évoquant par exemple des activités «structurées ", des situations de " jeux libres ", ou des " transitions ». Ils et elles mobilisent et mettent en œuvre des compétences spécifiques pour accomplir ces activités, dans des contextes caractérisés à la fois par une grande variabilité et un nombre important de personnes. Pour mieux comprendre les pratiques et les compétences professionnelles mobilisées dans le quotidien du travail, des travaux de recherche se sont intéressés à ces différents types d'activités (Filliettaz et Zogmal, 2020). Lors de la démarche ethnographique réalisée dans plusieurs structures d'accueil de la petite enfance en Ville de Genève et dans les entretiens de stage menés entre les éducatrices en formation et leurs référentes professionnelles, une autre forme d'activité éducative a été évoquée : la « relance ». Cette notion de relance, forgée par les praticien.ne.s issu-e.s du terrain, désigne l'ajustement situé d'une activité au contexte et aux conduites des participant.e.s. De cette manière, une relance désigne le processus au moyen duquel une intervention éducative est susceptible de remobiliser l'engagement des enfants dans une activité, au moment précis où les conditions de leur engagement sont évaluées comme moindres (par exemple cesser de s'intéresser à une activité, montrer un désintérêt pour des objets, etc.). Dès lors, une analyse focalisée sur la relance permet d'étudier l'imprévisibilité des pratiques éducatives et les compétences mobilisées par les éducatrices et éducateurs pour s'y ajuster.

Dans une perspective d'analyse des interactions, la description des pratiques de relance vise à mieux définir ce qu'est une relance. Comment s'accomplit-elle ? Comment les novices apprennent-ils ou elles à relancer une activité ? Afin de mieux comprendre les processus de transmission et d'apprentissage, il s'agit d'observer les relances telles qu'elles se produisent et se déploient dans le cours des activités éducatives, en prenant en compte la dimension collective des interactions entre les enfants, les enfants et les professionnel.le.s, les apprenant.e.s et les tuteurs ou tutrices.

Pour ce faire, cette contribution aborde en premier la notion de relance utilisée dans le champ de l'éducation de l'enfance (1). Pour étudier les pratiques de relance, une perspective interactionnelle est adoptée et présentée à la fois dans ses ingrédients théoriques (2) et dans ses spécificités méthodologiques (3). L'analyse empirique qui suit porte sur une activité de relance initiée par une éducatrice en formation et s'intéresse aux conduites interactionnelles de l'ensemble des participant.e.s (4). Cette analyse fait ressortir comment l'activité de relance modifie les modalités de participation des différents interactant.e.s et permet d'aboutir à une convergence de chacun.e vers une activité commune (5). En conclusion, nous interrogeons les apports d'une analyse interactionnelle du phénomène de relance pour mieux comprendre les enjeux de la formation en situation de travail (6). 


\section{La notion de relance}

Pour les éducatrices et éducateurs de l'enfance, la notion de relance désigne une action spécifique lors de la réalisation d'activités éducatives. Il s'agit de « relancer une activité ». Ceci ressort notamment des documents et outils méthodologiques mis à disposition par l'école professionnelle de l'éducation de l'enfance (ESEDE-CFPS, 2010a, 2010b, 2011) de Genève. La fiche de " conception d'un projet d'activité " mentionne la relance à deux reprises. En premier, la rubrique portant sur l'« organisation temporelle, spatiale et matérielle » utilise les termes de «matériel de relance ». Ensuite, la rubrique concernant la phase de déroulement de l'activité stipule : «Des relances verbales et/ou matérielles sont proposées pour élargir les possibilités d'action (si j’observe que... voilà ce que je peux...) ». Lors de la conception d'une activité, il est dès lors souvent prévu un matériel qui permette de relancer l'activité éducative planifiée, et d'anticiper des formes de relances verbales. Par ailleurs, la notion de relance apparaît dans la fiche d'" évaluation de l'activité réalisée " ESEDE-CFPS, 2010b) comme une des phases du déroulement des activités : "préparation, introduction, réalisation, relance, conclusion ». Ces fiches mobilisent la notion de relance pour désigner un matériel, des relances verbales ou des phases de l'activité. La relance de l'activité peut également constituer un des critères d'évaluation listés dans le « document pédagogique pour l'évaluation de l'examen professionnel pratique " qui mentionne la « gestion de la progression des situation éducatives".

Il est intéressant de noter que la relance fait partie des apports formatifs enseignés à l'école professionnelle mais n'apparaît pas en tant que telle dans le plan d'études cadre de la formation initiale de l'éducation de l'enfance ES (École supérieure). Ce cadre de référence aborde cependant l'animation des activités éducatives. Il situe l'animation comme un élément de la compétence professionnelle consistant à « soutenir le développement de l'enfant dans sa globalité ». Il stipule qu'il s'agit de savoir " proposer des activités adaptées aux compétences de l'enfant " (PEC, 2015). Dans d'autres documents de référence du champ professionnel, l'ajustement au rythme des jeunes enfants est également fortement valorisé afin de favoriser l'activité propre des enfants (Wustmann et Simoni, 2016).

Selon les fiches méthodologiques de l'école professionnelle, la relance contient des dimensions matérielles, interactionnelles et temporelles et forme une étape d'une activité éducative, prévue et planifiée. Sur un plan plus général, la relance vise à gérer « la progression des situations éducatives » et l'adaptation " aux compétences des enfants ». Certains ajustements peuvent être anticipés, tandis que d'autres s'avèrent nécessaire dans le cours des activités éducatives. Dès lors, les activités de relance peuvent également émerger au cours des interactions, «le moment venu».

En partant de ces différents éléments, la relance est ici définie comme l'introduction de propositions pour une réorientation de l'activité en cours, à travers la mise en œuvre de ressources variées (matérielles, verbales, etc.), en vue d'ajuster l'activité aux conduites et aux compétences des enfants et pour assurer sa progression. Les objectifs d'une relance peuvent être multiples et consister à faire redémarrer une activité ralentie ou interrompue, ou réparer et corriger une activité qui fonctionne mal. Une relance peut modifier légèrement l'activité initiale ou former la transition vers une nouvelle activité à part. La relance peut être planifiée ou émerger dans le déroulement de l'activité et s'adresser à un.e, à plusieurs ou à tou·te.s les enfants présent.e.s dans le contexte donné.

Les différents écrits et textes prescriptifs du champ professionnel ne permettent pas de cerner finement la relance en tant que pratique professionnelle. Comment une relance s'accomplit-elle ? Quels sont les enjeux de formation ? Comment les pratiques professionnelles mobilisées pour relancer une activité éducative et pour apprendre à relancer peuvent-elles être étudiées dans une perspective interactionnelle ?

\section{Une perspective interactionnelle sur les phénomènes de relance d'une activité éducative}

Dans le champ de la petite enfance, le travail éducatif est dans une large partie un travail interactionnel qui s'accomplit à travers une " co-activité de nature dialogale, dans laquelle un professionnel interagit avec un ou plusieurs interlocuteurs" (Pastré, 2011). De tels contextes professionnels présentent un caractère " discrétionnaire " (Pastré, 2011), au sens où ils produisent une obligation de résultats sans certitude des moyens permettant de les atteindre. L'imprévisibilité des conduites d'autrui, et notamment des jeunes enfants, demande aux éducatrices et aux éducateurs de s'ajuster continuellement aux particularités des situations rencontrées et de réorienter leurs actions (Filliettaz et Zogmal, 2020). Le travail ne peut pas se prescrire par des procédures opératoires ou 
se prévoir de façon détaillée dans ces contextes professionnels qui peuvent, dès lors, être désignés comme des « organisations de travail à prescription floue » (Faïta et Duc, 1996). Pour étudier les activités de travail, il s'agit dès lors d'aller au-delà des finalités éducatives et de s'intéresser au déroulement des pratiques réalisées dans le contexte du travail (Leplat et Hoc, 1983, p. 18). Dans les métiers éducatifs, une focale sur l'analyse des interactions permet d'étudier les pratiques dans le quotidien du travail, au-delà des discours prescriptifs.

L'ajustement des pratiques éducatives aux enfants et notamment la relance d'une activité se réalisent dans et à travers les interactions. Les interactions se caractérisent par les phénomènes d'interdépendance des participant.e.s (Sacks et al., 1974 ; Filliettaz, 2018). Pour s'ajuster l'un.e à l'autre, il s'agit de construire des significations partagées de ce qui se passe. Différentes perspectives théoriques permettent de relever l'importance de ces processus. Selon la notion d' " accountability » développée par Garfinkel (1967, 2007), les interactant.e.s se rendent mutuellement manifestes les éléments pertinents dans une situation d'interaction donnée. Ils et elles s'engagent dans des formes organisées d'actions, des ethnométhodes (Garfinkel, 1967, 2007), pour faire face aux «problèmes pratiques » rencontrés, et font ainsi advenir l'organisation d'un ordre social situé. Pour donner une visibilité à ce travail d'ajustement réciproque, une perspective d'analyse interactionnelle « endosse le point de vue des participants au moment où ils s'adressent mutuellement l'un à l'autre et où ils s'appliquent, 'à la deuxième personne' pourrait-on dire, à rendre leurs comportements mutuellement interprétables " (Filliettaz, 2018, p. 55). L'analyse de l'enchaînement des énoncés et des conduites interactionnelles permet de faire ressortir comment les participant.e.s rendent mutuellement visibles certaines significations et vers quels indices ils et elles s'orientent. L'aspect temporellement ordonné du langage verbal y contribue et les travaux en analyse conversationnelle (Sacks et al., 1974) se sont notamment intéressés à l'ordre séquentiel de l'interaction. De façon complémentaire à l'ordre séquentiel des interactions, un certain aspect de simultanéité se présente à travers la mobilisation de ressources sémiotiques variées. Les gestes, les regards, les positionnements, l'utilisation d'objets s'imbriquent avec le langage verbal, contribuent à faire émerger une signification et complètent, enrichissent ou modulent le sens des mots. Ces phénomènes de simultanéité ont été étudiés par les approches multimodales pour mieux comprendre le processus de "meaning-making» (Kress et al., 2001).

Dans le champ de la petite enfance, les activités éducatives se réalisent en présence d'un groupe d'enfants et de multiples professionnel.le.s. La dimension collective renforce les phénomènes d'ajustements réciproques, ainsi que certains aspects de simultanéité des interactions. Afin de mieux comprendre la relance d'une activité éducative menée dans un contexte collectif, il s'agit ici de présenter des perspectives théoriques portant sur les interactions avec de multiples participant.e.s. Dans de tels contextes, une activité unique, menée collectivement, peut regrouper l'ensemble des personnes présentes et constituer ainsi une interaction monofocalisée. Cependant, il est également possible que certaines activités se déroulent simultanément. Elles se réalisent alors dans un espace commun, mais s'accomplissent dans des foyers interactionnels distincts. Une telle situation d'interaction s'accomplit alors par des formes de polyfocalisations (Grosjean et Traverso, 1998). Parfois, les différentes activités s'effectuent de manière simultanée et forment une multiactivité menée dans un régime "parallèle» (Mondada, 2017, p. 340). À certains moments, plusieurs activités s'effectuent par des micro-alternances successives, par des focalisations qui passent d'une activité à une autre, pour revenir vers la première activité en cours. Une telle multiactivité se réalise alors dans un régime «imbriqué » (Mondada, 2017, p. 340).

Dans un contexte regroupant de multiples participant.e.s, les engagements dans les activités en cours se transforment par différentes façons. Une activité collective et monofocalisée peut donner lieu à une scission du cadre participatif, qui a été désignée par la notion de " schisme " (Egbert, 1997a, 1997b ; Sacks et al., 1974 ; Simmel, 1902 ; Traverso, 2012). Un schisme constitue une forme spécifique de multiactivité, qui se déroule dans un régime parallèle et qui se présente dans les situations réunissant au moins quatre participant·e.s, lorsqu'une interaction se scinde en deux foyers interactionnels. Dans chacun de ces foyers, une interaction se déroule en parallèle et comporte un ordre séquentiel. Ce qui se passe dans un foyer ne concurrence pas la progression de l'interaction dans l'autre foyer. Les travaux d'Egbert (1997a, 1997b) se sont intéressés aux " schismes conversationnels " lors des conversations pendant des repas en famille ou dans le cercle amical. Dans les situations de travail, l'activité langagière s'inscrit dans la réalisation de l'activité professionnelle et il est dès lors essentiel d'inclure les gestes et les conduites multimodales dans les analyses menées. L'importance des phénomènes multimodaux est soulignée par les travaux abordant la notion de "schisme interactionnel " (Markaki-Lothe et Filliettaz, 2017). Les transformations peuvent donc prendre la forme d'une scission des cadres participatifs. Elles peuvent également se manifester dans le sens contraire, lorsque plusieurs activités indépendantes convergent vers une activité unique et monofocalisée. La notion de convergence vise à désigner l'émergence d'une activité collective regroupant tous les participant·e.s, engagé.e.s préalablement dans plusieurs foyers actionnels ou interactionnels. 
L'analyse empirique cherche à décrire les liens entre le phénomène de la relance d'une activité éducative et les transformations des engagements interactionnels des participant.e.s. Elle vise également à étudier la dimension formative de l'activité de relance. Les éducatrices et éducateurs en formation apprennent, lors de leurs stages professionnels, à animer des situations éducatives. Les novices s'orientent vers l'accomplissement d'un travail éducatif, tout en étant accompagné.e.s par des praticien·ne·s expérimenté·e·s. La formation en contexte professionnel fait ressortir que les dimensions du travail et de la formation se présentent dans une seule et même situation. Les aspects éducatifs et formatifs s'imbriquent et constituent " une activité institutionnelle hybride " (Markaki-Lothe et Filliettaz, 2017, p. 81). Comment une analyse des interactions permet-elle d'aborder les pratiques de formation effectuées dans les situations de travail ?

\section{La démarche méthodologique}

Pour aborder les problématiques énoncées ci-dessus, une démarche de type vidéo-ethnographique a été adoptée, centrée sur les activités observables en situation. Le recueil de données s’inscrit dans le dispositif d'Études Supérieures au métier d'éducateur ou éducatrice de la petite enfance dans le canton de Genève, en conformité avec le Plan d'Études Cadres (PEC, 2015) édicté au niveau fédéral. Un partenariat de recherche a été négocié avec l'École Supérieure d'Éducatrices et Éducateurs de l'Enfance (ESEDE-CFPS) du canton de Genève et le Service de la petite enfance de la Ville de Genève. Au cours de leur formation, les étudiant.e.s réalisent plusieurs stages dans les institutions de la petite enfance, avec l'objectif de découvrir les pratiques professionnelles, de les expérimenter, et finalement de les maîtriser. Lors de ces stages, les étudiant·e-s sont placés sous la responsabilité de référent.e.s professionnel.le.s (voir Filliettaz et al., 2014).

Dans une démarche vidéo-ethnographique, le recueil des données porte sur une période de trois ans et concerne les stages de trois étudiantes, en première et en troisième année de formation. Pendant ces stages, les trois étudiantes sont accompagnées par six référentes professionnelles. La recherche suit les consignes éthiques de l'Université de Genève et s'effectue avec les consentements de l'ensemble des personnes présentes dans les situations observées. La durée globale des activités éducatives observées et enregistrées sous forme de films audio-vidéo se chiffre à $21 \mathrm{~h} 30$. Une pré-analyse a permis d'identifier une collection d'extraits de films portant sur des activités de relance, c'est-à-dire des réorientations de l'activité en cours réalisées par les stagiaires ou par les référentes professionnelles. Un tableau synoptique répartit ces extraits en différenciant, d'une part, les séquences initiées et menées par une des référentes professionnelles ou par une stagiaire, et d'autre part, en tenant compte du nombre d'enfants qui s'engagent progressivement dans l'activité de relance (voir Tableau 1).

\section{Tableau 1}

Répartition des relances observées selon la prise d'initiative et le nombre d'enfants concernés

\begin{tabular}{|l|l|l|l|}
\hline \multicolumn{2}{|l|}{ Initiation des relances } & \multicolumn{2}{|l|}{$\begin{array}{l}\text { Nombre d'enfants engagé·e·s dans l'activité de } \\
\text { relance }\end{array}$} \\
\cline { 2 - 4 } & Une partie des enfants & $\begin{array}{l}\text { Tous les enfants } \\
\text { présent·e·s }\end{array}$ \\
\hline Initiées par la référente & 4 situations & 3 situations \\
\hline $\begin{array}{l}\text { Initiées par la } \\
\text { stagiaire }\end{array}$ & $\begin{array}{l}\text { Hors présence de la } \\
\text { référente }\end{array}$ & 1 situation & - \\
\cline { 2 - 4 } & $\begin{array}{l}\text { Animées } \\
\text { conjointement avec la } \\
\text { référente }\end{array}$ & 5 situations & 1 situation \\
\cline { 2 - 4 } & $\begin{array}{l}\text { Observées par la } \\
\text { référente }\end{array}$ & 1 situation & 2 situations \\
\hline
\end{tabular}

Afin de pouvoir étudier la dimension formative et les processus d'apprentissage de la stagiaire, le choix de la séquence analysée porte sur une des relances initiées et menées par une stagiaire, sous l'observation de la référente professionnelle. Il s'agit de relances non planifiées et qui émergent dans le cours des interactions. Deux de ces activités de relance amènent progressivement la participation de tou.te.s les enfants à une activité collective. La séquence finalement retenue se déroule en plusieurs phases et permet ainsi d'étudier les pratiques professionnelles mises en œuvre par la stagiaire et les pratiques de formation déployées par la référente professionnelle. 
La séquence choisie est issue d'un stage de première année de formation. Léducatrice en formation est accompagnée par une référente professionnelle qui a une longue expérience du travail auprès de jeunes enfants. La relance survient lors d'une situation de jeux libres qui suit une activité éducative, préparée et animée par la stagiaire. Ce moment de jeux libres regroupe six enfants âgé.e.s d'environ deux ans. Il est mis en place en attendant l'heure du repas de midi et n'a pas été planifié auparavant.

Le film de l'activité de relance analysée est d'une durée de 1.03 minutes et il est reparti en six extraits, afin de faciliter son analyse. Les extraits sont délimités lorsqu'un·e ou plusieurs des interactant.e.s modifient leurs façons de participer à l'activité en cours, en passant d'un·e interlocuteur ou interlocutrice à un.e autre, par exemple. L'activité observée se déroule dans la salle de jeux des enfants (voir figure 2).

\section{Figure 1}

Situation de jeux libres

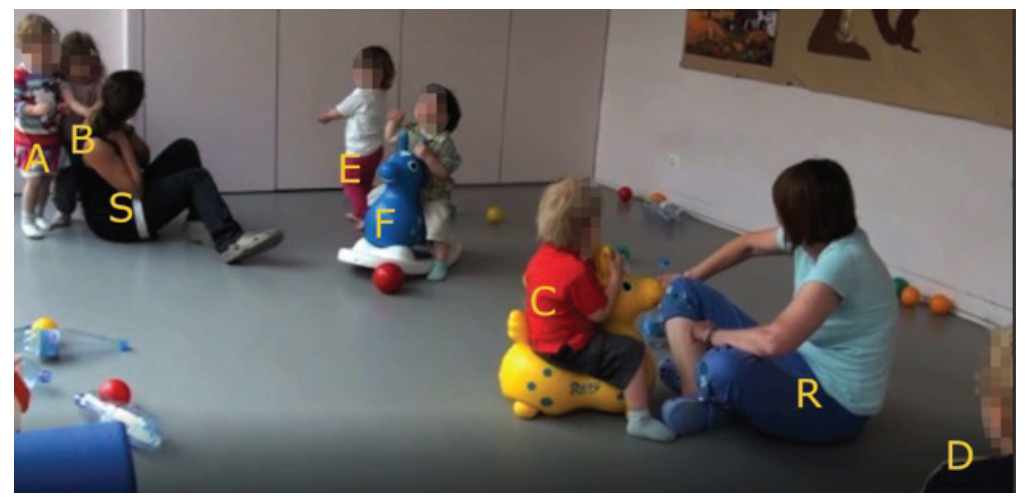

Au début de la séquence analysée, la stagiaire $(S)$ et la référente professionnelle $(R)$ s’installent par terre, après avoir mis à disposition du matériel de jeu complémentaire (des chevaux à bascule). Les balles et les bouteilles qui ont servi pour l'activité précédente sont encore dispersées dans la salle. Les enfants (âgé·e.s d'environ deux ans), Anna (A), Béatrice (B), Claude (C), David (D), Emma (E) et Frédéric (F) s'engagent alors dans de multiples activités, individuellement ou par groupes de deux ou trois.

Les conventions de transcription utilisées pour présenter les données empiriques sont explicitées en annexe.

\section{L'animation d'une situation de « jeux libres " et la relance d'une activité éducative}

\subsection{La situation initiale}

Pendant ce moment de jeux libres, plusieurs activités se déroulent en même temps. Dans le foyer interactionnel orienté vers la stagiaire, les deux enfants A et B tapent avec leurs mains sur le haut du dos de la stagiaire. Celle-ci se tient la nuque et se retourne vers les deux enfants. Elle les regarde pendant un court instant et reste immobile. La stagiaire rend manifeste qu'elle observe les enfants. Simultanément, la référente professionnelle interagit avec $\mathrm{C}$ et répond à ses sollicitations. À plusieurs reprises, elle pose une bouteille par terre, et $\mathrm{C}$ essaie de l'attraper en avançant avec son cheval à bascule. Chacune des professionnelles s'engage dans un foyer interactionnel distinct. En même temps, D se déplace sur un cheval de bascule et s'engage dans une activité de façon individuelle. E et $\mathrm{F}$ s'orientent l'un.e vers l'autre. E initie des conduites d'un jeu symbolique qui se mettra en place progressivement dans les extraits 1 à 4. Dans ce premier extrait, E tend ses mains, formant un creux, à $F$.

Extrait 1

\begin{tabular}{|c|c|c|c|c|c|c|}
\hline & & Foyer I & Foyer II & Foyer III & & Foyer IV \\
\hline & $A+B$ & tapent avec la main & C encore/ & D se déplace, & $E$ & tend ses mains \\
\hline & s & $\begin{array}{l}\text { surle dos de } S \\
\text { se tient la nuque }\end{array}$ & $\begin{array}{l}\mathrm{R} \text { encore / } \\
\text { prends une bouteille }\end{array}$ & $\begin{array}{l}\text { assis sur un } \\
\text { cheval en }\end{array}$ & F & $\begin{array}{l}\text { vers } F \\
\text { regarde } E\end{array}$ \\
\hline & $s$ & $\begin{array}{l}\text { se retourne et } \\
\text { regarde } A \text { et } B\end{array}$ & $\begin{array}{l}\text { et la pose } \\
\mathrm{C} \text { voilà }\end{array}$ & plastique & $E$ & $\begin{array}{l}\text { se déplace et fait } \\
\text { le tourde } F\end{array}$ \\
\hline
\end{tabular}


Une scission de la participation dans de multiples activités parallèles s'est mise en place, qui peut être désignée comme une multiactivité polyfocalisée. La répartition en plusieurs foyers interactionnels ou actionnels peut être schématisée comme sur la Figure 3.

\section{Figure 3}

Répartition en plusieurs foyers interactionnels ou actionnels

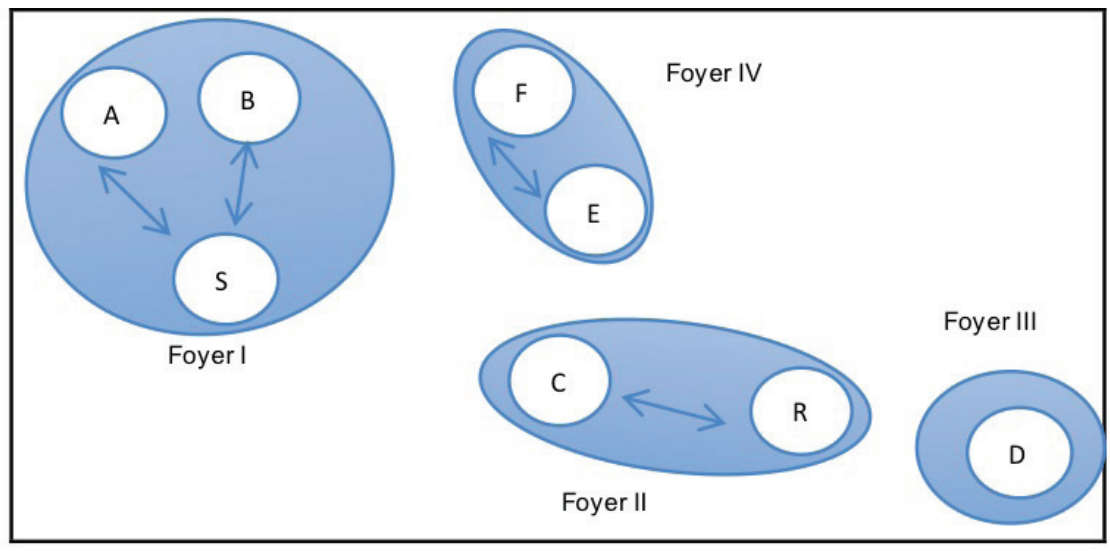

Deux enfants (A, B) tapent sur le dos de la stagiaire (foyer I), un enfant (C) interagit avec la référente (foyer II), tandis qu'un autre enfant (D) s'engage dans une conduite individuelle (foyer III), et deux enfants (E, F) interagissent ensemble (foyer IV).

\subsection{L'initiation d'une activité de relance orientée vers deux enfants}

La situation initiale d'une multiactivité polyfocalisée perdure dans l'extrait suivant, tout en se transformant. La stagiaire, après avoir observé $\mathrm{A}$ et $\mathrm{B}$, commence à proposer une nouvelle activité, en s'adressant exclusivement aux deux enfants en question. Ainsi, la stagiaire réoriente les conduites de A et B, qui sont en train de taper sur son dos. Simultanément, la référente professionnelle n'interagit plus uniquement avec C, mais également avec D. Il subsiste donc trois foyers interactionnels distincts.

\begin{tabular}{|c|c|c|c|c|c|c|}
\hline $\begin{array}{l}6 \\
7\end{array}$ & $\mathrm{~S}$ & $\begin{array}{l}\text { on va:: ta::per.. } \\
\text { [sur le pied }\{\end{array}$ & $\mathrm{C}$ & $\begin{array}{l}\text { saute avec son cheval et } \\
\text { avance vers la bouteille }\end{array}$ & $\mathrm{E}$ & $\begin{array}{l}\text { avance vers la paroi } \\
\text { regarde vers } S\end{array}$ \\
\hline 8 & & [S repousse la main d'A et la & & & & \\
\hline $\begin{array}{l}9 \\
10\end{array}$ & & $\begin{array}{l}\text { regarde } \\
\text { \{S tape sur ses deux pieds }\end{array}$ & $\mathrm{R}$ & $\begin{array}{l}\text { regarde } D \text { qui s'avance } \\
\text { vers elle }\end{array}$ & & \\
\hline 11 & A & \{regarde le mouvement de $S$ & & & & \\
\hline $\begin{array}{l}12 \\
13\end{array}$ & S & $\begin{array}{l}\text { )sur tes pieds Anna } \\
\text { Jlève ses deux mains en }\end{array}$ & $\mathrm{R}$ & regarde $S$ & $\mathrm{~F}$ & regarde $E$ \\
\hline $\begin{array}{l}14 \\
15\end{array}$ & & $\begin{array}{l}\text { hauteur en regardant les pieds } \\
\text { de } S\end{array}$ & & & $\mathrm{~F}$ & saute avec son \\
\hline 16 & $\mathrm{~S}$ & $\S$ sur les tiens & & & & cheval \\
\hline $\begin{array}{l}17 \\
18\end{array}$ & $\begin{array}{l}\text { A } \\
\text { S }\end{array}$ & $\begin{array}{l}\text { § tape sur le pied de } S \\
\& \text { ils sont où }\end{array}$ & $\mathrm{C}$ & $\begin{array}{l}\text { se penche et prend une } \\
\text { bouteille }\end{array}$ & & \\
\hline 19 & & \&S regarde $A$ & & & & \\
\hline $\begin{array}{l}20 \\
21\end{array}$ & A & $\begin{array}{l}\text { \& continue à taper surle pied } \\
\text { de S }\end{array}$ & & & & \\
\hline 22 & $\mathrm{~S}$ & ils sont où & & & & \\
\hline 23 & & $=$ les tiens à toi & & & & \\
\hline 24 & A & $=A$ s'assied par terre & & & & \\
\hline $\begin{array}{l}25 \\
27\end{array}$ & B & $\begin{array}{l}=B \text { s'assied par terre et tape sur } \\
\text { les pieds de } S\end{array}$ & & & & \\
\hline 27 & $\mathrm{~S}$ & \$Béatrice aussi. & & & & \\
\hline $\begin{array}{l}28 \\
29\end{array}$ & & $\begin{array}{l}\text { \$S regarde } B \\
\text { Eils sont où tes pieds à toi }\end{array}$ & D & $\begin{array}{l}\text { se penche brusquement en } \\
\text { avant }\end{array}$ & $\mathrm{E}$ & $\begin{array}{l}\text { s'approche de } F \\
\text { avec les mains en }\end{array}$ \\
\hline 30 & A & Etape sur ses pieds & $\mathrm{R}$ & regarde $D$ & & creux \\
\hline $\begin{array}{l}31 \\
32\end{array}$ & B & $\begin{array}{l}\text { Earrête de taper sur les pieds } \\
\text { de S }\end{array}$ & & & & \\
\hline 33 & $\mathrm{~S}$ & ils sont où les tiens & & & & \\
\hline
\end{tabular}


Dans le foyer d'activité focalisé sur la stagiaire, celle-ci propose par un acte directif une réorientation des conduites des enfants. A et B sont en train de taper sur le dos de la stagiaire. La stagiaire énonce qu'il s'agit de taper sur le pied (1. 6). Simultanément, elle repousse légèrement la main d'A (1. 8-9). Ensuite, la stagiaire se met à taper avec ses deux mains sur ses pieds. A observe le mouvement de la stagiaire et regarde les pieds de la stagiaire. À la suite de ce regard, la stagiaire précise son énoncé précédent et complète : "sur tes pieds Anna " (1. 12). Malgré cette précision, A se met à taper sur le pied de la stagiaire (1. 17). La stagiaire interpelle alors A à plusieurs reprises pour lui indiquer qu'il s'agit de taper sur ses propres pieds (1. 18, 22, 23). La stagiaire focalise son regard sur A et continue en même temps de faire un mouvement rythmique et amplifié en tapant sur ses pieds. Par suite de ces différentes conduites, A arrête de taper sur le pied de la stagiaire et s'assied par terre (1. 24). Pendant ce temps de quelques secondes, la deuxième enfant, B, observe. Elle imite les conduites d'A lorsque celle-ci s'assied et tape sur les pieds de la stagiaire (1. 25). La stagiaire répète ses énoncés préalables ("ils sont où tes pieds à toi ", 1.29), en les adressant individuellement à B. La réorientation de l'activité s'accomplit à travers les énoncés directifs de la stagiaire, ses gestes, ses conduites de monstration et la variation des adressages. Les répétitions et la mobilisation simultanée de nombreuses ressources multimodales amènent à une structuration de l'activité en cours et constituent l'initiation d'une activité de relance orientée vers les deux enfants en question. Une action de réparation des conduites des enfants qui "dérangent " la stagiaire se transforme en activité de relance qui se déploie dans le temps jusqu’à ce que les deux enfants s'y alignent.

Pendant ce temps, les interactions dans les autres foyers d'activité continuent à se dérouler en parallèle. Seule la référente professionnelle (1. 11) et $\mathrm{E}$ (1. 7) regarde brièvement vers la stagiaire, ce qui montre une certaine porosité entre les foyers d'activité. Par ailleurs, la référente professionnelle continue à observer $\mathrm{C}$, ainsi que $\mathrm{D}$ qui s'approche d'elle. E et F s'orientent toujours l'un.e vers l'autre, lorsque E s'approche de $\mathrm{F}$ en tenant ses mains en creux (1. 28-30).

Dans cet extrait, la situation de multiactivité subsiste. La stagiaire a initié une relance qui lui a permis de réorienter les conduites de $\mathrm{A}$ et $\mathrm{B}$ vers une activité menée conjointement par les deux enfants et la stagiaire. Cette émergence d'une activité collective dans un des foyers interactionnels va attirer progressivement l'attention des autres interactant.e.s, dans les extraits suivants.

\section{3. Élargissement progressif de L'activité de relance vers l'ensemble des participant-e-s}

Dans l'extrait suivant (3), l'activité de relance attire l'attention de C.

\section{Extrait 3}

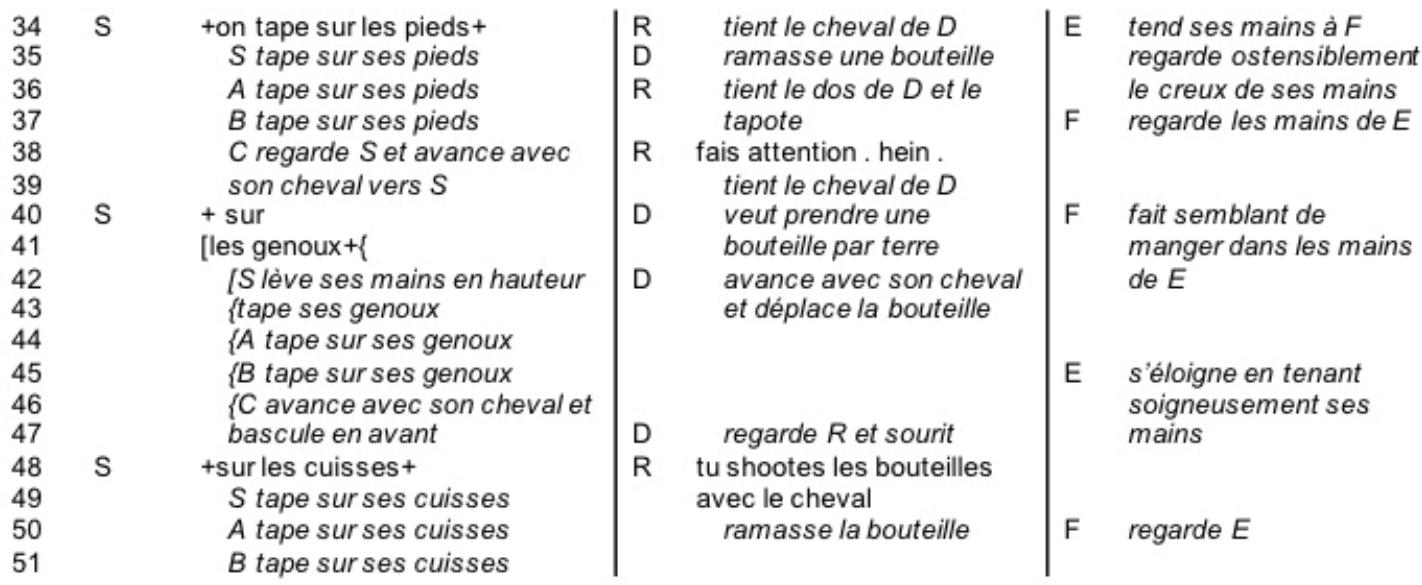

Dans cet extrait, l'activité de taper sur les différentes parties du corps, animée par la stagiaire, se déroule de façon ritualisée. La stagiaire indique les parties du corps concernées, en accompagnant ses verbalisations par les gestes correspondants. La stagiaire, A et B tapent sur leurs pieds. C se met à observer ce mouvement et s'approche. La stagiaire énonce qu'il s'agit de taper sur les genoux (1. 40-41), et sur les cuisses (1. 48). Simultanément à ses énoncés, la stagiaire tape sur les parties de son corps qu'elle vient de nommer. Les conduites sont devenues routinisées. A et $\mathrm{B}$ continuent à imiter les mouvements de la stagiaire en reprenant rapidement ses différentes propositions. $\mathrm{C}$ avance encore plus en direction de la stagiaire et de A et B, tout en restant assis sur son cheval à bascule (1. 46-47). 
De façon parallèle, deux autres activités se déroulent dans des foyers interactionnels distincts. Dans le premier de ces foyers, la référente professionnelle tient le cheval de D (1. 34) pour éviter qu'il ne bascule et lui dit de faire attention (1. 38). Pendant ce temps, D essaie de prendre une bouteille qui est posée par terre (1. 40-43). Par son mouvement, son cheval à bascule pousse la bouteille et la déplace (1. 43). D regarde alors la référente professionnelle et lui sourit. Celle-ci rend alors manifeste ce qu'elle a observé : " tu shootes les bouteilles avec le cheval " (1. 49-50). La référente professionnelle s’oriente vers les conduites de D, en assurant sa sécurité ainsi qu'en mettant en mot ce qu'elle observe de ses conduites. Elle s'aligne aux initiatives prises par l'enfant. Dans l'autre foyer interactionnel, E tend ses mains à F. F se penche au-dessus des mains de E et fait semblant de manger dans ses mains (1. 40-42). E s'éloigne ensuite, en tenant soigneusement ses mains, pour aller « rechercher à manger " (1. 45-47).

Dans le quatrième extrait, l'accomplissement de l'activité de relance attire un autre enfant.

\section{Extrait 4}

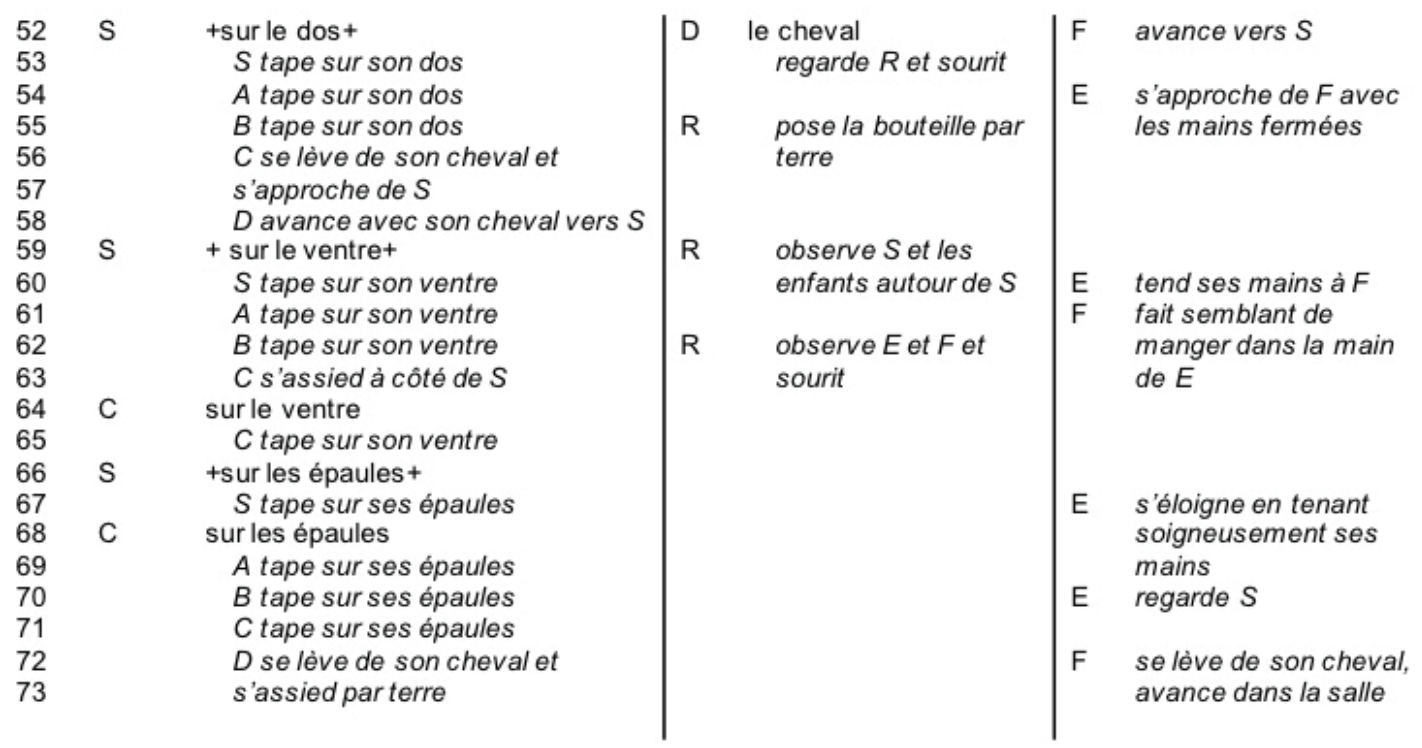

La stagiaire continue l'animation de l'activité de relance, tandis que $\mathrm{A}, \mathrm{B}$ et $\mathrm{C}$ imitent ses conduites. $\mathrm{C}$ répète également les énoncés de la stagiaire $(1.64,68)$. Les alignements des enfants aux consignes de la stagiaire sont immédiats et les quatre participant.e.s (S, A, B, C) s'engagent dans un même mouvement rythmique. Pendant cet extrait, la stagiaire se focalise sur A et B. Elle oriente son regard clairement vers les deux enfants en question et ajuste son rythme à leur rythme. Elle ne regarde ni $\mathrm{C}$ ni $\mathrm{D}$ qui se sont approchés.

$\mathrm{D}$ continue d'interagir avec la référente, mais réoriente son attention. Il se déplace vers la stagiaire (1. 58) et s'assied par terre (1. 72-73). E et F continuent leur activité, tout en rendant visible qu'il et elle s'orientent en même temps vers l'activité menée par la stagiaire. F se déplace en direction de la stagiaire (1. 52), tandis que $\mathrm{E}$ regarde la stagiaire (1. 70). Les trois enfants (D, E, F) s'engagent de façon imbriquée dans deux activités simultanées. La référente professionnelle observe que $\mathrm{D}$ a réorienté son attention vers la nouvelle activité de relance. Elle pose alors la bouteille qu'elle tient dans la main et reste immobile (1. 55). Elle observe E et F et sourit lorsque F fait semblant de manger dans la main de E (1. 62). Elle suspend toute activité propre. Cette immobilité, son regard orienté vers la stagiaire et les enfants qui l'entourent, et ensuite vers $\mathrm{E}$ et $\mathrm{F}$ montrent qu'elle a adopté une posture d'observation (1. 59-64). À la suite de la réorientation de D et de la suspension de l'activité de la référente professionnelle, seulement deux foyers interactionnels subsistent (cf. Figure 4). 
Figure 4

Posture d'observation de R orientée vers deux foyers interactionnels

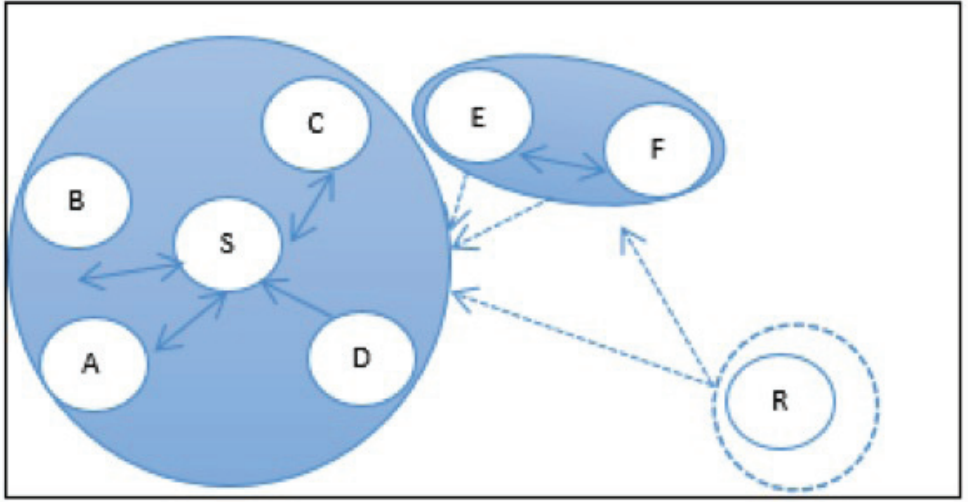

\subsection{L'émergence d'une activité commune}

Dans l'extrait suivant (5), l'ensemble des enfants s'orientent progressivement vers l'activité de relance mise en place par la stagiaire.

\section{Extrait 5}

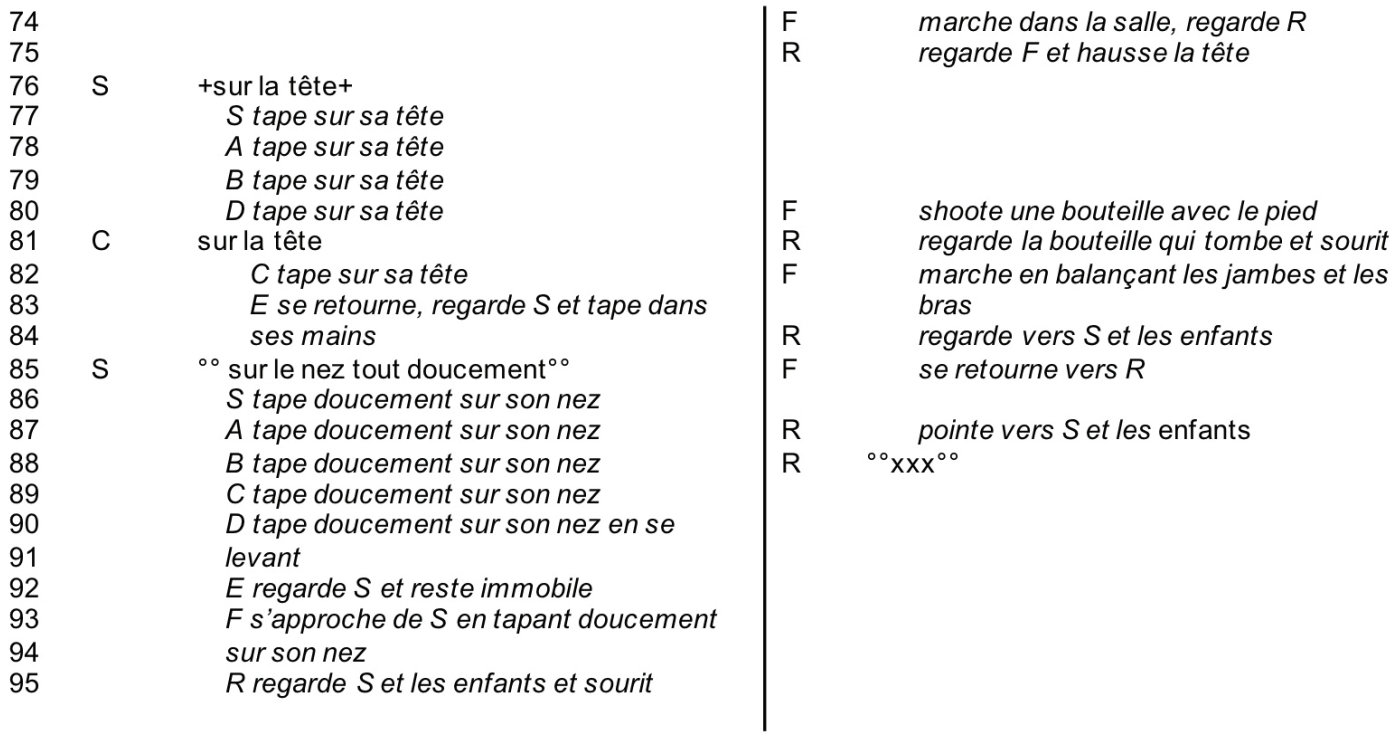

Dans cet extrait, l'activité propre de E et F s'estompe progressivement. Les deux enfants ne s'orientent plus l'un.e vers l'autre. E observe la stagiaire (1. 83-84 et 93), tandis que F commence à se déplacer dans la salle (l. 74), à donner un coup de pied dans une bouteille (1. 80), et à marcher avec des mouvements de balancements (1. 82-83). Son regard ne se focalise pas et ses déplacements, peu linéaires, semblent montrer une sorte de " désœuvrement ». La référente observe $\mathrm{F}(1.75,81)$ et lui sourit $(1.81)$. Lorsque F la regarde (1. 85), la référente professionnelle le regarde, pointe vers la stagiaire et les enfants et s'adresse à voix basse à l'enfant (1. 87-88, voir schéma 5, ci-dessous). À travers ces différentes ressources interactionnelles, elle initie une focalisation de l'attention de F sur l'activité menée par la stagiaire. F s'approche alors de la stagiaire et des enfants qui se trouvent autour d'elle. Tout en avançant dans la salle, il commence à taper doucement sur son nez, en imitant l'activité collective en cours (1. 93-94). 


\section{Figure 5}

Geste de pointage de la référente professionnelle vers l'activité de relance

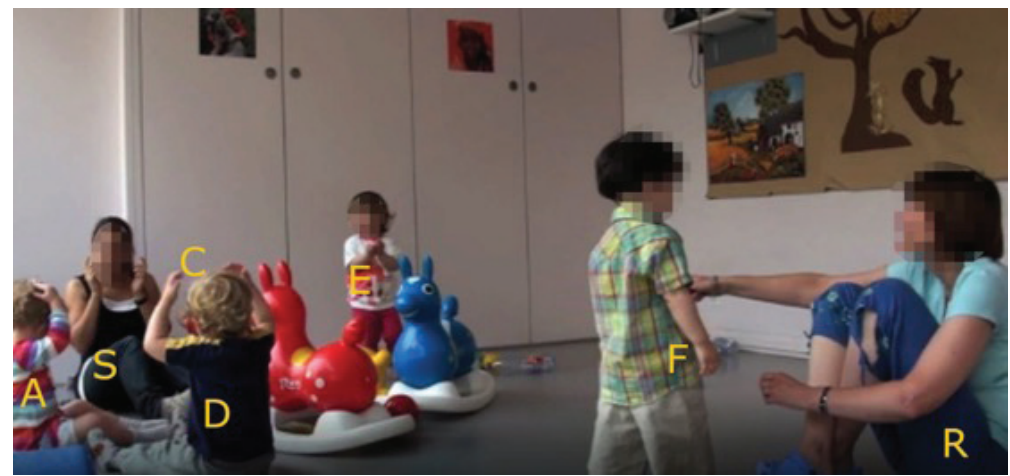

Dans l'extrait suivant, une activité collective et focalisée se met en place, regroupant l'ensemble des participant.e.s.

\section{Extrait 6}

\begin{tabular}{|c|c|c|}
\hline 96 & $\mathrm{~S}$ & ${ }^{\circ}$ sur le menton ${ }^{\circ}$ \\
\hline 97 & & S tape sur son menton \\
\hline 98 & & A tape sur son menton \\
\hline 99 & & B tape sur son menton \\
\hline 100 & & $D$ se met debout et tape sur son menton \\
\hline 101 & & E regarde $S$ et reste immobile \\
\hline 102 & & F tape sur son menton en levant la tête \\
\hline 103 & $\mathrm{C}$ & hein/ \\
\hline 104 & $\mathrm{~F}$ & avance \\
\hline 105 & D & pousse légèrement $F$, puis il recule \\
\hline 106 & S & tape doucement sur son menton et regarde $A$ \\
\hline 107 & & A rit \\
\hline 108 & & $D$ rit et s'assied sur le cheval qu'il avait avant \\
\hline 109 & & E s'approche du cheval que $F$ avait laissé \\
\hline 110 & S & et les joues. elles sont où les joues / \\
\hline 111 & C & 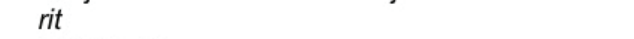 \\
\hline 112 & $\mathrm{~S}$ & regarde $C$ \\
\hline 113 & & F se déplace vers $S$ \\
\hline 114 & & E touche le cheval avec son pied \\
\hline 115 & $\mathrm{~S}$ & elles sont où les joues / \\
\hline 116 & & C rit \\
\hline 117 & & A rit \\
\hline 118 & A & + là/+ \\
\hline 119 & & A tape sur ses joues \\
\hline 120 & & $F$ se tient les joues en regardant $S$ \\
\hline 121 & & $S$ regarde $A$ \\
\hline 122 & $\mathrm{~S}$ & elles sont làl \\
\hline 123 & & S se tape les joues \\
\hline 124 & & A se tape les joues \\
\hline 125 & & B se tape les joues \\
\hline 126 & & C se tape les joues \\
\hline 127 & & $D$ se tape les joues \\
\hline 128 & & F se tape les joues \\
\hline 129 & & E monte sur le cheval en regardant $S$ \\
\hline 130 & & $R$ regarde $S$ et les enfants et retient un sourire \\
\hline
\end{tabular}

Tout en s'approchant de la stagiaire et des autres enfants, F imite les conduites proposées par la stagiaire, en tapant sur son menton. La nouvelle consigne de la stagiaire n'est pas tout de suite comprise par C (" hein », 1 . 103) qui ne s'aligne pas immédiatement. En même temps, $D$ se lève et se met debout. F s'approche de $D$ (l. 105). Celui-ci le pousse (1. 105), avant de le contourner pour monter sur le cheval qu'il avait avant (l. 108). Tandis qu'auparavant, l'alignement des enfants à l'activité de relance était immédiat, une légère perturbation se manifeste. La stagiaire continue à taper doucement sur son menton et focalise son regard sur A (1. 106). Celle-ci se met à rire (1. 107), imitée par D (1. 108). E s'approche du cheval utilisé par F auparavant (1. 109), le 
touche (1. 114), avant de monter dessus (1. 129). Le désalignement d'une partie des enfants amène la stagiaire à modifier ses énoncés et le cours de l'activité. Elle ne nomme plus une partie du corps, mais pose une question : " et les joues, elles sont où les joues" (1. 110). Elle oriente son regard vers A. C se met à rire (1. 111). C'est la première fois depuis l'initiation de cette activité de relance que la stagiaire réoriente son regard et ne se focalise plus sur A et B. Elle regarde C (1. 112). Elle répète sa question (1. 115). C et A continuent à rire $(1.116,117)$. La stagiaire regarde à nouveau A qui lui répond à voix haute (" là », 1. 118), tout en tapant sur les joues. La stagiaire s'aligne et se met à taper sur ses joues, tout en énonçant : " elles sont là " (1. 122). Tou·te·s les enfants se mettent à taper sur leurs joues, à part $\mathrm{E}$ qui monte sur un cheval à bascule, tout en regardant attentivement S. L'activité collective mise en place se poursuivra encore pendant un moment, en impliquant l'ensemble des enfants présent.e.s. Pendant toute la durée de cet extrait, la référente professionnelle observe l'activité en cours.

\section{La relance comme moteur d'un processus de convergence}

L'analyse montre que l'activité de relance se construit de façon située, dans et par le déroulement des interactions. Dans la situation analysée, la relance intervient dans le cours d'une situation complexe avec de multiples participant.e.s, à travers un processus de convergence progressif. À partir de multiples foyers d'activité, elle regroupe tou·te.s les participant.e.s. Une activité collective et monofocalisée se met en place. Ces changements des modalités de participation sont accomplis à travers les conduites de l'ensemble des personnes présentes, et notamment par les alignements progressifs des enfants. Le cours de l'interaction se déploie dans un agencement séquentiellement ordonné dans le temps et de façon simultanée. Dans la figure 6, les différentes activités dans des foyers interactionnels distincts se déroulent d'abord en parallèle, avant de se rejoindre dans une activité commune.

\section{Figure 6}

Changements des modalités de participation

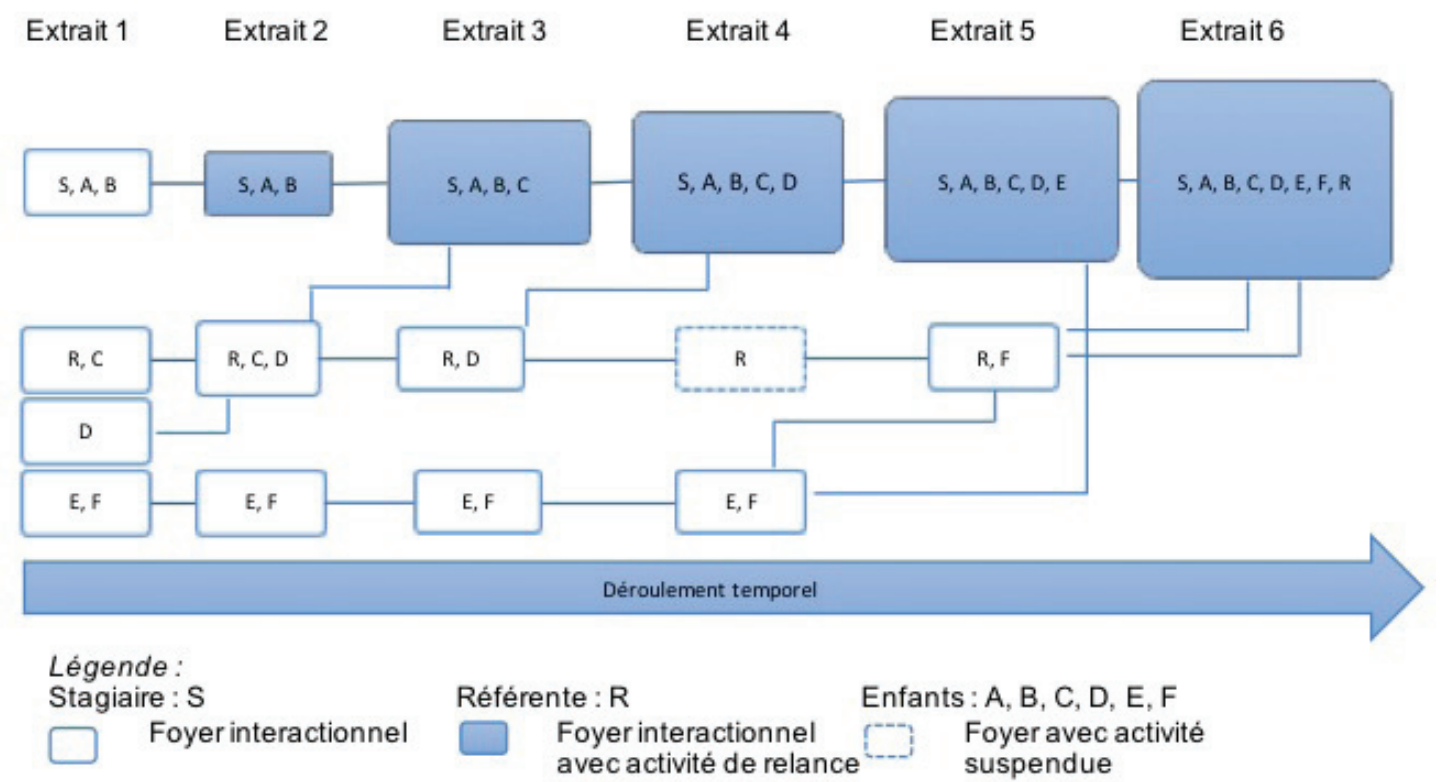

Réorientations des participants vers un autre foyer interactionnel

Pendant toute la séquence, la stagiaire reste focalisée sur l'activité de relance consistant à taper sur les parties du corps. Elle s'ajuste au rythme d'engagements des enfants, en modulant la prosodie, les gestes et les conduites. Cette mise en visibilité d'une focalisation exclusive renforce l'aspect structurée et séquentiellement ordonnée de son activité. La référente, quant à elle, s'engage de façon imbriquée dans les différents foyers d'activité. Elle interagit avec les enfants qui se trouvent autour d'elle, tout en observant l'activité de la stagiaire et celle de E et 
F. La référente tient ainsi compte de l'ensemble des activités en cours. L'oscillation de ses focalisations permet d'instaurer des interactions avec les enfants et de réorienter leur attention vers l'activité animée par la stagiaire. La posture de la référente professionnelle et ses conduites soutiennent la co-construction d'une activité de relance. Lorsque les enfants focalisent leur attention vers la stagiaire et les enfants qui l'entourent, la référente professionnelle suspend ses conduites interactionnelles, s'abstient de bouger, regarde de façon soutenue vers les enfants et la stagiaire et observe ainsi de façon publiquement manifeste ce qui se passe. Elle intervient également activement pour orienter l'attention de F vers l'activité en cours et pour l'encourager de la rejoindre. D'une façon qui semble très discrète lors d'une analyse rapide de la situation, la référente professionnelle renforce la visibilité de la relance. Les conduites de la référente offrent une opportunité à la stagiaire de se centrer sur l'activité de relance initiée et lui permettent de maintenir sa focalisation sans perturbation. En ce qui concerne les enfants, $\mathrm{A}$ et $\mathrm{B}$ se focalisent dès le début de cette séquence sur la stagiaire. Les autres enfants s'orientent brièvement ou plus longuement sur plusieurs activités de façon imbriquée, avant de rejoindre l'activité de relance.

Dans la situation analysée, un enjeu de la relance constitue à reconstruire, dans des conditions fluides, une activité collective susceptible d'attirer et de maintenir l'attention des enfants et présentant un intérêt éducatif. L'initiation de la relance intervient dans une situation de polyfocalisation. Les enfants sont engagé.e.s, de manière individuelle ou en petits groupes, dans plusieurs activités indépendantes. La mise en place d'une activité collective, rythmique et ritualisée amplifie la visibilité de ce qui se passe, et fait converger l'ensemble des enfants vers une focalisation partagée. Une activité collective, commune à l'ensemble des participant.e.s à l'interaction, se met en place.

\section{Le phénomène de relance et les enjeux de la formation en situation de travail}

L'analyse des activités de relance à partir de traces filmiques et dans une perspective interactionnelle permet de mettre au jour les aspects de complexité et de dynamique de la relance. La convergence vers une seule activité collective renforce l'imbrication des aspects formatifs et des aspects liés à la dimension du travail, qui forment ainsi une situation d'hybridité.

En ce qui concerne les aspects éducatifs, la relance en tant que processus de convergence permet aux enfants de s'engager dans une activité conjointe, d'attirer et de maintenir leur intérêt et leur focalisation sur l'activité en cours. Laccomplissement progressif favorise l'ajustement aux rythmes de compréhension et d'engagement de chacun.e des enfants. La relance contribue ainsi à orienter les conduites des enfants sans imposer. La complexité du déroulement interactionnel et la richesse des opportunités éducatives constituent des arguments pour inclure la relance dans les catégorisations des activités éducatives et de s'y intéresser, au même titre qu'aux activités structurées, aux jeux libres ou aux transitions.

Sur le plan formatif, les phénomènes de scissions de la participation et la convergence progressive vers une activité collective permettent de favoriser les prises d'initiatives et l'accomplissement de pratiques éducatives par la stagiaire. Ils contribuent également à un accompagnement de la référente professionnelle qui n’entre pas en compétition dans la mobilisation de l'attention des enfants. L'action de la référente, à travers une posture d'observation, constitue bien une attitude de "laisser faire ", selon les catégories développées par Kunégel (2012), mais comme cet auteur le relève justement, " contrairement à ce que nous avons cru longtemps, il ne s'agit pas toujours d'un désengagement des maittres» (p. 4). La référente professionnelle se focalise sur l'activité mise en place par la stagiaire, elle lui donne une visibilité accrue et une légitimité rendue manifeste pour l'ensemble des personnes présentes. En s'abstenant d'agir, elle offre une opportunité de formation.

La compréhension du phénomène de la relance permet de mieux reconnaître les pratiques tutorales parfois discrètes, de soutenir l'analyse réflexive portant sur les activités de relance et rendre compte de la complexité des situations d'interactions regroupant de multiples participant.e.s. Un regard analytique sur la relance permet de montrer la diversité des modalités de participation des enfants et des professionnel.le.s dans le cours des activités éducatives. Relancer une activité ne s'accomplit pas par l'action individuelle d'une éducatrice ou d'un éducateur, mais nécessite une co-construction collective. Une meilleure compréhension des phénomènes interactionnels et de leur ordre séquentiel et simultané et des différentes modalités de participation constitue une base pour analyser le cours des interactions et s'y ajuster. Le développement des compétences professionnelles implique ainsi une prise en compte de la dynamique collective des situations d'interactions.

En ce qui concerne la formation initiale, une perspective interactionnelle peut amener un changement de regard sur l'activité de relance et sur sa dimension collective. Dans la séquence analysée, ce sont les conduites 
des enfants, à travers leurs reprises et leurs transformations, qui offrent des ressources à la stagiaire pour initier une relance. Leurs alignements et désalignements influencent fortement la progression de l'activité collective. Par ailleurs, les conduites de la référente professionnelle contribuent à accomplir une convergence de l'ensemble des participant.e.s vers une activité commune. Des analyses interactionnelles sur de telles situations de relance peuvent favoriser une plus grande focale sur l'aspect dynamique et collectif des situations éducatives. Ceci permet de dépasser une délimitation du regard sur la relation entre un·e professionnel.le et un·e enfant individuel.le et d'ouvrir la réflexion sur la dimension collective des interactions.

\title{
Annexes : Conventions de transcription
}

I
।
+
ACCent
(incertain)
XX
:
-
.......
souligné
((commentaire))

\author{
Intonation montante \\ Intonation descendante \\ Diminution du volume de la voix \\ Augmentation du volume de la voix \\ Enoncé accompagné d'un rire dans la voix
}

Accentuation

$<$ mhmm > Régulateurs verbaux produits par le destinataire

Segments dont la transcription est incertaine

Segments intranscriptibles

Allongements syllabiques

Troncations

Pauses de durée variable

Chevauchements dans les prises de parole

Commentaire du transcripteur, relatif à des conduites gestuelles ou des actions non verbales

\section{Références bibliographiques}

Egbert, M. M. (1997a). Schisming : The collaborative transformation from a single conversation to multiple conversations. Research on Language et Social Interaction, 30(1), 1-51.

Egbert, M. (1997b). Some interactional achievements of other-initiated repair in multiperson conversation. Journal of Pragmatics, 27, 611-634.

ESEDE-CFPS. (2010a). Conception d'un projet d'activité. ESEDE-CFPS.

ESEDE-CFPS. (2010b). Évaluation de l'activité réalisée. ESEDE-CFPS.

ESEDE-CFPS. (2011). Document pédagogique pour l'évaluation de l'examen professionnel pratique. ESEDE-CFPS.

Faïta, D., et Duc, M. (1996). Savoir-faire d'encadrement et prescription floue. Dans J. Girin et M. Grosjean (Dir.), La transgression des règles au travail (p. 51-82). L'Harmattan.

Filliettaz, L. (2018). Interactions verbales et recherche en éducation. Principes, méthodes et outils d'analyse. Carnets des sciences de l'éducation.

Filliettaz, L., Rémery, V., et Trébert, D. (2014). Relation tutorale et configurations de participation à l'interaction : le cas de la formation professionnelle des éducatrices et éducateurs de l'enfance. Activités, 11(1), 22-46.

Filliettaz, L., et Zogmal, M. (Dir.). (2020). Mobiliser et développer des compétences interactionnelles en situation de travail éducatif. Editions Octarès.

Garfinkel, H. (1967). Studies in Ethnomethodology. Prentice-Hall.

Garfinkel, A. (2007). Recherches en ethnométhodologie. Presses universitaires de France.

Grosjean, M., et Traverso, V. (1998). Les cadres participatifs dans les polylogues : problèmes méthodologiques. Dans F. Cabasino (Dir.), Du dialogue au polylogue (p. 51-67). CISU.

Kress, G., Jewitt, C., Ogborn, J., et Charalampos, T., (2001) Multimodal teaching and learning. The rhetorics of the science classroom. Continuum.

Kunégel, P. (2012, 3-6 juillet). Analyse des pratiques des mâ̂tres d'apprentissage en situation de travail. [Communication orale]. Biennale internationale de l'éducation, de la formation et des pratiques professionnelles, Paris, France. https://halshs.archivesouvertes.fr/halshs-00867025/document

Leplat, J., et Hoc, J.-M. (1983). Tâche et activité dans l'analyse psychologique des situations, Cahiers de psychologie cognitive, 3, 49-64. Repris dans J. Leplat (Dir.). (1992). L'analyse du travail en psychologie ergonomique (p. 47-60, Tome I). Octarès.

Markaki-Lothe, V., et Filliettaz, L. (2017). "Comment faire les remarques dans l'effervescence du travail? » : une analyse interactionnelle des scissions des cadres participatifs dans la formation professionnelle des éducateurs de la petite enfance. Travaux Neuchâtelois de Linguistique, 67, 79-102.

Mondada, L. (2017). Le défi de la multimodalité en interaction. Revue française de linguistique appliquée, XXII(2), 71-87.

Pastré, P. (2011). La didactique professionnelle. Presses universitaires de France.

Plan d'études cadres. (PEC). (2015). Éducatrice de l'enfance ES, Éducateur de l'enfance ES. SPAS/OrTraS. 
Sacks, H., Schegloff, E., et Jefferson, G. (1974). A simplest systematics for the Organization of Turn-Taking for Conversation. Language, 50, 696-735.

Simmel, G. (1902). The number of members as determining the sociological form of the group. American Journal of Sociology, $8(2), 158-196$.

Traverso, V. (2012). Ad hoc-interpreting in multilingual work meetings. Dans C. Baraldi et L. Gavioli (Dir.), Coordinating participation in dialogue interpreting (p. 149-176). John Benjamins.

Wustmann, C., et Simoni, H. (2016). Cadre d'orientation pour la formation, l'accueil et l'éducation de la petite enfance en Suisse (3e éd.). Commission suisse pour l'Unesco et Réseau d'accueil extrafamilial.

Mots-clés : Formation professionnelle ; analyse interactionnelle ; éducation de l'enfance ; dimension collective ; interactions multi-participants

\section{Lernen, eine pädagogische Aktivität neu einzuführen/ einzuüben: die berufliche Ausbildung im Bereich der frühkindlichen Bildung}

\section{Zusammenfassung}

Dieser Artikel befasst sich mit der Identifizierung und Entwicklung professioneller Kompetenzen im Bereich der frühkindlichen Bildung. Er analysiert Interaktionen in der alltäglichen Berufspraxis und interessiert sich dafür, wie eine pädagogischen Aktivität neu eingeführt und gelernt werden kann. Zuerst wird der Begriff der Neuorientierung diskutiert, bevor die Konzepte einer interaktionellen Analyse vorgestellt werden. In der empirischen Analyse wird insbesondere die Art der Partizipation der verschiedenen Teilnehmer untersucht, welche progressiv eine gemeinsame Aktivität umsetzen. Die Analyse einer Ausbildungssituation gibt Aufschluss über Zusammenhänge zwischen dem Phänomen der neuen Einführung einer pädagogischen Aktivität und den Herausforderungen der Berufsausbildung.

Schlagworte: Berufsausbildung; Interaktionsanalyse; Frühkindliche Bildung; Betreuung und Erziehung; Kollektive Dimension; Multi-partizipative Interaktionen

\section{Imparare a "rilanciare» un'attività educativa: La formazione professionale sul posto di lavoro nel contesto della prima infanzia}

\section{Riassunto}

Questo articolo si concentra sull'identificazione e lo sviluppo di competenze professionali nel campo della prima infanzia. Da una prospettiva interazionale, un'analisi dettagliata affronta le pratiche reali mobilitate per imparare a rilanciare un'attività educativa. L'articolo discute il concetto del rilancio, utilizzato dagli operatori del settore, prima di presentare l'approccio di analisi interattiva adottato. L'analisi empirica descrive un'attività di rilancio e studia le modalità di partecipazione dei diversi interlocutori che gradualmente convergono verso un'attività comune. L'attenzione ad una situazione di stage professionale aiuta a far luce sui legami tra il fenomeno del rilancio e le sfide della formazione in una situazione lavorativa.

Parole chiave: Formazione professionale; analisi interattiva; prima infanzia; dimensione collettiva; interazioni multi-partecipanti 


\section{Learning how to "relaunch" an educational activity : Vocational training in the field of Early Childhood Education}

\section{Summary}

This article focuses on the identification and development of professional competences in the field of Early childhood education. Following an interactional perspective on training and work, a detailed analysis addresses the real practices deployed to learn how to relaunch an educational activity. First, the article discusses the notion of "relaunching" (relance), used by practitioners in the field. It then presents the theoretical perspective of interactional analysis. An empirical case study follows, consisting in a fine-grained description of a relaunching activity conducted by an intern student in collaboration with her mentor. The analysis focuses on the dynamics of participation amongst different participants, who gradually converge towards a joint activity. The concluding section sheds light on the links between the phenomenon of relaunching an educational activity and the challenges of vocational education and training.

Keywords: Vocational training; Interactional analysis; early childhood education; collective dimension; multiparticipant interactions

Marianne Zogmal, Dre, Collaboratrice scientifique, Formation d'adultes. Marianne Zogmal s'intéresse aux liens entre interactions, travail et formation ainsi qu'aux apports d'une perspective interactionnelle pour la formation professionnelle dans les métiers de l'humain.

Université de Genève, Interaction et Formation, Case postale, 1211 Genève

E-Mail: Marianne.Zogmal@unige.ch

Laurent Filliettaz est professeur ordinaire dans le domaine Formation des adultes, langage et travail. Ses recherches portent sur les interactions en situation de travail et de formation.

Université de Genève, Interaction et Formation, Case postale, 1211 Genève

E-Mail: Laurent.Filliettaz@unige.ch

Vassiliki Markaki-Lothe, Dre, Maitre de conférence. Enseignante-chercheure à l'UGA, elle s'intéresse aux processus d'apprentissage et aux conditions locales et situées dans lesquelles s'élaborent les interactions formatives entre expert.e.s et novices.

Laboratoire de Recherche sur les Apprentissages en Contexte (LaRAC), Université Grenoble-Alpes, CS 40700, F-38058 Grenoble cedex 9

E-Mail: vasiliki.markaki-lothe@univ-grenoble-alpes.fr 